Escuela de Ciencias Sociales y Humanidades, UNED, Costa Rica https://revistas.uned.ac.cr/index.php/espiga ISSN: 1409-4002・e-ISSN: 2215-454X

\title{
Uso del tiempo de las mujeres rurales jefas de hogar en Costa Rica
}

\section{Use of time by rural women who are heads of household in Costa Rica}

\section{La gestion du temps des femmes rurales chefs de famille au Costa Rica}

Ángel Jesús Porras-Solís * https://orcid.org/0000-0002-9394-1912

Recibido: 19 de mayo de 2021 • Aceptado: 13 de agosto de 2021

* Licenciado en Economía por la Universidad Nacional (UNA), de Costa Rica. Máster en Técnicas Estadísticas por la Universidad de Santiago Compostela, de España. Académico e investigador en la Sede Regional Brunca de la UNA. Es tutor en la Cátedra de Estadística de la Escuela de Administración de la Universidad Estatal a Distancia, de Costa Rica. Correo: angel.porras.solis@una.ac.cr 


\section{Resumen}

En Costa Rica, más de una tercera parte de los hogares rurales tienen una mujer como jefa de hogar; la mayoría de ellas se debe incorporar al mercado laboral, con limitados niveles de escolaridad, empleos poco calificados y de baja remuneración, para dar sostén económico a su hogar. Aunque hoy se reconoce una mayor participación de las mujeres en las actividades económicas del medio rural, los datos de la Encuesta Nacional de Uso del Tiempo (2017) muestran que, por un amplio margen, siguen soportando la mayor carga de trabajo doméstico no remunerado. Justamente, el objetivo de este artículo es dar a conocer, mediante datos concretos, la realidad de las mujeres rurales jefas de hogar en cuanto a la forma en que distribuyen su tiempo en actividades de trabajo remunerado y no remunerado, así como cuantificar su posición respecto de los hombres rurales jefes de hogar.

Palabras clave: Género, mujeres rurales, mujeres jefas de hogar, trabajo doméstico no remunerado.

\section{Abstract}

In Costa Rica, more than one third of rural households have a woman as head of household; most of them must join the labor market, with limited levels of schooling, to low-skill and low pay jobs, in order to provide economic support for their home. Although today a greater participation of women in rural economic activities is recognized, data from the National Survey of Time Use (2017) show that, by a wide margin, they continue to bear the greatest burden of unpaid domestic work. Precisely, the objective of this article is to make known, through concrete data, the reality of rural women heads of household in terms of the way in which they distribute their time in paid and unpaid work activities, as well as to quantify their position with respect to rural men heads of household.

Keywords: Gender, rural women, women heads of household, unpaid domestic work.

\section{Résumé}

Au Costa Rica, plus d'un tiers des foyers ruraux ont une femme comme chef de famille; la plupart d'elles doivent s'intégrer au marché du travail avec des niveaux de scolarisation limités ainsi que des emplois peu qualifiés et mal rémunérés afin d'assurer un soutien économique à leurs familles. Dans l'actualité on reconnaît qu'il y a une plus grande participation des femmes dans les activités économiques du milieu rural, cependant les données de l'Enquête Nationale de l'Usage du Temps (2017) montrent, avec une grande marge, que les femmes continuent à supporter la plus grande charge du travail domestique non rémunéré. C'est pourquoi cet article vise à faire connaître la réalité des femmes chefs de famille concernant la gestion du temps à travers des données précises. Le but est de mettre en évidence la distribution du temps qu'elles font entre les activités du travail rémunéré et non rémunéré, ainsi que quantifier leur position par rapport à celle des hommes ruraux chefs de famille.

Mots-clés: Genre, femmes chefs de famille, travail domestique non rémunéré. 


\section{Introducción}

Muchos de los estudios realizados en América Latina indican que, en el contexto de los nuevos espacios rurales, se han venido generando nuevas alternativas para las mujeres, reflejado a través de mejores opciones de inserción al mercado laboral y el acceso a la educación, en especial para las mujeres jefas de hogar. De igual forma, se evidencia un aumento en el porcentaje de mujeres jefas de hogar, como resultado de una diversidad de factores económicos y culturales que van desde las dificultades de los hombres para obtener mayores ingresos y mantenerse como proveedores únicos, el aumento en la escolaridad de las mujeres y su participación laboral, hasta la ruptura de uniones conyugales no satisfactorias o violentas ${ }^{1}$.

No obstante, como señalan Sandoval y González², las mujeres todavía siguen concentrando las actividades domésticas del hogar, al encargarse de la mayoría de las tareas de «reproducción» social, de las labores de cuidado infantil y de enfermos, así como del mantenimiento del hogar, lo cual es invisibilizado en las estadísticas oficiales por considerarse que no genera valor al no producir bienes y servicios que puedan transarse en el mercado. Las cifras disponibles sobre la situación laboral de las mujeres en el área rural muestran que las brechas de género de la participación y la tasa de ocupación son mucho más acentuadas que en las zonas urbanas, lo cual tiene su explicación en las mayores dificultades que tienen las mujeres rurales para conseguir un empleo respecto de los hombres, así como a la invisibilidad de las trabajadoras rurales que cumplen actividades familiares no remuneradas 0 productoras para el autoconsumo, no son captadas como ocupadas en las encuestas ${ }^{3}$.

En este contexto, los datos sobre uso del tiempo se constituye en una importante herramienta para comprender el modo en que la participación de la mujer en la fuerza laboral remunerada conduce al crecimiento de la actividad del mercado para reemplazar actividades no remuneradas del hogar 0 , alternativamente, la forma en que la transmisión de servicios de atención de las

\footnotetext{
1 Navarro-Ochoa, Angélica, «¿Mujeres proveedoras y jefas de familia?, Nuevas realidades rurales en localidades de la región zamorana», La ventana, Revista de estudios de género 4, n. 31 (2010): 139-171, acceso: 25 de enero de 2021, http://www.scielo.org.mx/scielo.php?script=sci_arttext\&pid=S140594362010000100007\&lng=es\&tlng=es

2 Irma Sandoval-Carvajal y Lidia María González-Vega, «Estimación del valor económico del trabajo no remunerado en Costa Rica. Resultados e ilustración metodológica», Estudios Demográficos y Urbanos 30, n. ${ }^{\circ}$ 3 (2015): 691-724, acceso: 02 de febrero de 2021, https://estudiosdemograficosyurbanos.colmex.mx/ index.php/edu/article/view/1497/1490

${ }^{3}$ Alicia Bárcena, «Mujeres rurales y Agenda 2030 en América Latina y el Caribe: una mirada desde el mercado de trabajo», en: Luchadoras: mujeres rurales en el mundo: 28 voces autorizadas, 21-26 (San José: IICA, 2018), acceso: 15 de enero de 2021, https://repositorio.iica.int/bitstream/handle/11324/7221/

BVE18040310e. pdf?sequence=1\&isAllowed=y
} 
instancias públicas a la comunidad significa un aumento de la actividad no remunerada a cargo de trabajadores «invisibles» 4 .

En el caso particular de Costa Rica, los resultados de la Encuesta Nacional de Uso del Tiempo 2017 (ENUT 2017) muestran que las brechas de género en el uso del tiempo entre hombres y mujeres siguen estando mediadas por la cantidad de tiempo empleado en la realización del trabajo doméstico no remunerado, donde son las mujeres quienes continúan asumiendo la mayor parte de estas tareas. Por su parte, las cargas globales diferenciadas por sexo, también reflejan realidades muy diferentes para mujeres y hombres en el mercado de trabajo, los hombres tienen una menor carga de trabajo doméstico no remunerado y cuentan con mayores oportunidades para incorporarse y mantenerse en el mercado laboral, capacitarse y lograr mejores condiciones laborales.

Por lo tanto, tomando en cuenta las anteriores consideraciones y utilizando como fuente de información la ENUT 2017, el principal objetivo del presente artículo es conocer y cuantificar la forma en que este grupo de mujeres rurales jefas de hogar distribuye su tiempo entre trabajar con el fin de generar ingresos para dar sostén económico a sus familias, llevar a cabo labores de trabajo doméstico no remunerado, estudiar y otras actividades, incluyendo las relacionadas con su tiempo libre. Lo anterior se complementa con la descripción de sus principales características sociodemográficas, así como un análisis comparativo con respecto de los hombres rurales en su misma condición de jefes de hogar, en términos del tiempo dedicado a las actividades de no trabajo y de trabajo, así como la carga global de trabajo. Es importante considerar que los datos utilizados son previos al inicio de la pandemia y que, en definitiva, el uso del tiempo se ha modificado a raíz de este fenómeno.

\section{Desarrollo}

Características sociodemográficas de las mujeres rurales jefas de hogar en Costa Rica

En el presente apartado se describen las principales características sociodemográficas de las mujeres rurales jefas de hogar en Costa Rica a luz de los resultados de la ENUT 2017. Dicha encuesta considera tamaño muestral total efectivo de 1473 jefes de hogar rurales, donde la muestra de mujeres jefas de

\footnotetext{
${ }^{4}$ Marilyn Waring, «Política y estrategia de provisión de la atención de salud en la región de la OPS y medición del trabajo no remunerado», en: La economía invisible y las desigualdades de género. La importancia de medir y valorar el trabajo no remunerado, 245-286 (Washington D.C.: OPS, 2008), acceso: 15 de enero de 2021, https://iris.paho.org/bitstream/handle/10665.2/6034/LA\%20ECONOMIA\%20INVISIBLE.pdf? sequence=1\&isAllowed=y
} 
hogar considerada para el presente trabajo es de 537 (36,5\%), que corresponde a una población total estimada de 168950 mujeres, una vez aplicado el respectivo factor de expansión.

Como se detalla en el cuadro 1 , cerca del $60 \%$ de las mujeres rurales tiene entre 20 y 55 años de edad, menos del $2 \%$ tiene 19 años o menos y un $36,69 \%$ tiene 55 años 0 más. Por su parte, cerca del $45 \%$ se encuentra separada o soltera. El porcentaje de mujeres en unión libre o casada representan 32,9\%. Asimismo, se puede destacar un importante porcentaje de mujeres viudas y divorciadas, que en forma conjunta suman casi un $22 \%$ del total.

En cuanto al nivel de instrucción, el $82,35 \%$ de las mujeres rurales jefas de hogar cuenta con primaria y secundaria incompleta. Solamente el 7,53 \% tiene secundaria completa y el 10,12 \% cuenta con algún grado de educación superior. Un aspecto por destacar es que el 93,59 \% de estas mujeres, al

\section{Cuadro 1}

Costa Rica: Mujeres rurales jefas de hogar. Principales características sociodemográficas

\begin{tabular}{|c|c|c|}
\hline Grupo de edad & Cantidad & Porcentaje \\
\hline 19 años o menos & 1913 & 1,13 \\
\hline De 20 a 35 años & 48404 & 28,65 \\
\hline De 36 a 54 años & 56652 & 33,53 \\
\hline De 55 y más años & 61981 & 36,69 \\
\hline \multicolumn{3}{|l|}{ Estado conyugal } \\
\hline En unión libre o juntada & 29481 & 17,45 \\
\hline Casada & 26096 & 15,45 \\
\hline Divorciada & 13534 & 8,01 \\
\hline Separada & 39633 & 23,46 \\
\hline Viuda & 23451 & 13,88 \\
\hline Soltera & 36755 & 21,75 \\
\hline \multicolumn{3}{|l|}{ Nivel de instrucción } \\
\hline $\begin{array}{l}\text { Primaria incompleta y } \\
\text { menos }\end{array}$ & 46511 & 27,53 \\
\hline $\begin{array}{l}\text { Primaria completa y } \\
\text { secundaria incompleta }\end{array}$ & 92623 & 54,82 \\
\hline Secundaria completa & 12719 & 7,53 \\
\hline Superior & 17097 & 10,12 \\
\hline
\end{tabular}

Fuente: INEC, Costa Rica. Encuesta Nacional de Uso del Tiempo, 2017. 
momento de la encuesta, no asiste a ningún tipo de institución educativa y, de las que sí asiste, lo hace a principalmente a educación no regular, universidad o educación abierta.

Las que indican no asistir a algún tipo de instrucción, no lo hacen por alguna condición de embarazo o matrimonio, tienen que llevar alguna labor de cuido de personas, deben trabajar o se les dificulta el estudio. Por su parte, otras manifiestan que no asisten por no tener la posibilidad de pagar los estudios, tener que ayudar a oficios domésticos o les falta ganar alguna prueba o examen para continuar.

Sin duda, estos bajos niveles de escolaridad e imposibilidad de continuar estudios redundan en empleos poco calificados y de bajas remuneraciones. Efectivamente, si se examinan las condiciones laborales, casi el $80 \%$ tienen ocupaciones poco calificadas.

En cuanto a la posición en el empleo, el 36,17 \% lleva a cabo su actividad laboral en un negocio o actividad propia, un $38,88 \%$ como empleada de una persona, empresa o institución. Por su parte, un 23,01 \% trabaja como empleada de casas particulares. De las que reportan un ingreso salarial monetario en el empleo principal, en promedio reciben mensualmente $\mathbb{L} 199$ 226,54. No obstante, es importante indicar que el $50 \%$ recibe un salario inferior a $\mathbb{l} 120000$ por mes y solamente el $25 \%$ supera los $\mathbb{L} 259800$ mensuales. Si se toman en consideración los ingresos percibidos a través de un empleo secundario, solamente un $5,55 \%$ lo reporta, con un promedio de ingreso equivalente a $\mathbb{L} 30$ 240,31 mensuales. Por otra parte, respecto a los ingresos percibidos por concepto de transferencias (jubilaciones, pensiones alimenticias o subsidios estatales o dinero de otras personas), en promedio reciben $\mathbb{l} 142627,77$ por mes, donde cerca del $65 \%$ percibe un monto inferior a $\mathbb{Q} 100000$ mensuales. Un

\section{Cuadro 2}

Costa Rica: Mujeres rurales jefas de hogar. Asistencia a educación formal

\section{Asistencia}

No asiste

Escuela

Colegio

Parauniversitaria

Universidad

Educación abierta en institutos

para presentar exámenes del MEP

Educación no regular

Total

Fuente: INEC, Costa Rica. Encuesta Nacional de Uso del Tiempo, 2017.

\section{Cantidad Porcentaje}

\begin{tabular}{|c|c|}
\hline 158122 & 93,59 \\
\hline 860 & 0,51 \\
\hline 1285 & 0,76 \\
\hline 563 & 0,33 \\
\hline 2593 & 1,53 \\
\hline 2474 & 1,46 \\
\hline
\end{tabular}

3053

1,81

168950 
aspecto por destacar es que el $71,11 \%$ de estas mujeres debe sostener económicamente su hogar a través de los ingresos en mención.

Uso del tiempo según tipo de actividad

En el presente apartado se analiza el uso del tiempo de las mujeres rurales jefas de hogar a través de la tasa de participación y el tiempo efectivo dedicado según el tipo de actividad. Siguiendo la metodología del INEC , las actividades se agrupan en dos categorías: actividades de trabajo y no trabajo. Las actividades de trabajo comprenden las relacionadas con el trabajo doméstico no remunerado, trabajo de autoconsumo, trabajo de apoyo a otros hogares y a la comunidad, así como el trabajo remunerado. Por su parte, las actividades de no trabajo considera aquellas de formación, tiempo libre y necesidades personales.

\section{Cuadro 3}

Costa Rica: Mujeres rurales jefas de hogar. Motivos por las que no asisten a educación formal

\begin{tabular}{|c|c|c|}
\hline Motivo & Cantidad & Porcentaje \\
\hline Tiene que trabajar & 1979 & 20,51 \\
\hline $\begin{array}{l}\text { Tiene que cuidar niños(as), } \\
\text { personas adultas mayores u otras } \\
\text { personas }\end{array}$ & 2010 & 20,83 \\
\hline $\begin{array}{l}\text { Tiene que ayudar en oficios } \\
\text { domésticos }\end{array}$ & 572 & 5,93 \\
\hline No puede pagar los estudios & 663 & 6,87 \\
\hline $\begin{array}{l}\text { Problemas de acceso al sistema } \\
\text { escolar }\end{array}$ & 176 & 1,82 \\
\hline Le cuesta el estudio & 1445 & 14,97 \\
\hline $\begin{array}{l}\text { No está interesado(a) en el } \\
\text { aprendizaje formal }\end{array}$ & 186 & 1,93 \\
\hline Embarazo o matrimonio & 2278 & 23,60 \\
\hline $\begin{array}{l}\text { Falta ganar prueba del MEP/ } \\
\text { exámenes de admisión }\end{array}$ & 342 & 3,54 \\
\hline Total & 9651 & 100 \\
\hline
\end{tabular}

Fuente: INEC, Costa Rica. Encuesta Nacional de Uso del Tiempo, 2017.

5 INEC, Encuesta Nacional de Uso del Tiempo 2017: Resultados generales (San José, INEC, 2018), 38, acceso: 12 de julio de 2021, https://www.inec.cr/sites/default/files/documetos-biblioteca-virtual/reenut2017.pdf 
Actividades de trabajo

Al estudiar las actividades que conforman el trabajo doméstico no remunerado, en el cuadro 5 se observa que las cuatro actividades en las cuales las mujeres tienen mayor participación se relacionan con la limpieza y mantenimiento de la vivienda, la preparación y el servicio de alimentos y bebidas, la limpieza y el cuidado de la ropa y calzado. Le siguen otras actividades como la gerencia y

\section{Cuadro 4}

Costa Rica: Mujeres rurales jefas de hogar. Ocupación y posición en el empleo principal

\begin{tabular}{|l|c|c|}
\hline Ocupación & Cantidad & Porcentaje \\
\hline Profesionales científicos e intelectuales & 4584 & 5,10 \\
\hline Técnicas y profesionales de nivel medio & 2085 & 2,32 \\
\hline $\begin{array}{l}\text { Personal de apoyo administrativo } \\
\text { Trabajadoras de los servicios y vendedoras de } \\
\text { comercios y mercados }\end{array}$ & 35494 & 3,39 \\
\hline $\begin{array}{l}\text { Agricultoras y trabajadoras calificadas } \\
\text { agropecuarios, forestales y pesqueros }\end{array}$ & 5797 & 6,46 \\
\hline $\begin{array}{l}\text { Oficiales, operarias y artesanas de artes } \\
\text { mecánicas y de otros oficios }\end{array}$ & 2749 & 3,06 \\
\hline $\begin{array}{l}\text { Operadoras de instalaciones y máquinas y } \\
\text { ensambladores }\end{array}$ & 2713 & 3,02 \\
\hline $\begin{array}{l}\text { Ocupaciones elementales } \\
\text { Total }\end{array}$ & 35136 & 39,13 \\
\hline $\begin{array}{l}\text { Posición } \\
\text { Un negocio o actividad propia }\end{array}$ & $\mathbf{8 9} \mathbf{8 0 4}$ & $\mathbf{1 0 0}$ \\
\hline $\begin{array}{l}\text { Como empleada o empleado de una } \\
\text { persona/empresa o institución }\end{array}$ & 32485 & 36,17 \\
\hline $\begin{array}{l}\text { Como empleada o empleado de casas } \\
\text { particulares }\end{array}$ & 34920 & 38,88 \\
\hline $\begin{array}{l}\text { Como ayuda a una persona familiar o conocido } \\
\text { que tiene un negocio o actividad propia }\end{array}$ & 1210 & 1,35 \\
\hline $\begin{array}{l}\text { Como ayuda a una persona o familiar conocido } \\
\text { que trabaja para un patrón o empresa }\end{array}$ & 523 & 0,58 \\
\hline Total & $\mathbf{8 9} \mathbf{8 0 4}$ & $\mathbf{1 0 0}$ \\
\hline
\end{tabular}

Fuente: INEC, Costa Rica. Encuesta Nacional de Uso del Tiempo, 2017. 


\section{Cuadro 5}

Costa Rica: Mujeres rurales jefas de hogar. Actividades de trabajo. Tasa de participación y tiempo efectivo promedio

\begin{tabular}{|c|c|c|}
\hline Actividades de trabajo & $\begin{array}{l}\text { Tasa de } \\
\text { participa- } \\
\text { ción }\end{array}$ & $\begin{array}{l}\text { Tiempo } \\
\text { efectivo } \\
\text { promedio }\end{array}$ \\
\hline Trabajo doméstico no remunerado & 99,79 & 42:38 \\
\hline $\begin{array}{l}\text { Preparación y servicio de alimentos y } \\
\text { bebidas }\end{array}$ & 97,05 & $16: 47$ \\
\hline Limpieza y mantenimiento de la vivienda & 97,95 & $11: 44$ \\
\hline $\begin{array}{l}\text { Construcción, mantenimiento y } \\
\text { reparaciones menores de la casa y } \\
\text { vehículo }\end{array}$ & 14,67 & $1: 22$ \\
\hline $\begin{array}{l}\text { Limpieza, cuidado y confecciones de } \\
\text { ropa y calzado }\end{array}$ & 93,72 & $5: 22$ \\
\hline Compras del hogar & 57,54 & $2: 29$ \\
\hline Gerencia y administración del hogar & 60,40 & $1: 59$ \\
\hline $\begin{array}{l}\text { Cuidado de niños y niñas menores de } 12 \\
\text { años }\end{array}$ & 41,42 & $35: 44$ \\
\hline $\begin{array}{l}\text { Cuidado y apoyo a otros miembros del } \\
\text { hogar de } 12 \text { años y más (que no sean } \\
\text { totalmente dependiente) }\end{array}$ & 17,43 & $16: 47$ \\
\hline $\begin{array}{l}\text { Cuidado de personas integrantes del } \\
\text { hogar totalmente dependientes }\end{array}$ & 3,21 & $14: 02$ \\
\hline Trabajo de autoconsumo & 40,01 & $4: 41$ \\
\hline $\begin{array}{l}\text { Trabajo no remunerado de apoyo a } \\
\text { otros hogares y a la comunidad }\end{array}$ & 28,66 & $5: 14$ \\
\hline Trabajo remunerado & 54,91 & 33:55 \\
\hline Trabajo en ocupación principal & 51,94 & $31: 20$ \\
\hline Trabajo ocupación secundaria & 5,50 & $10: 31$ \\
\hline Trasladarse a ocupación principal & 40,37 & $3: 32$ \\
\hline Trasladarse a ocupación secundaria & 4,80 & $1: 29$ \\
\hline
\end{tabular}

Fuente: INEC, Costa Rica. Encuesta Nacional de Uso del Tiempo, 2017. 
administración del hogar, las compras del hogar y el cuidado de niños y niñas menores de 12 años.

En cuanto al tiempo efectivo promedio ${ }^{6}$ que dedican a estas a actividades de trabajo doméstico no remunerado, las que más horas registran son el cuido de menores y de otros miembros de la familia. A estas actividades de cuido, le siguen la preparación y servicio de alimentos y bebidas, así como la limpieza y mantenimiento de la vivienda. El promedio de tiempo dedicado al trabajo doméstico no remunerado es cercano a las 43 horas.

En relación con el trabajo de autoconsumo y trabajo no remunerado de apoyo a otros hogares y a la comunidad, las cifras correspondientes a la tasa de participación y al promedio de horas dedicadas a la semana son relativamente bajas. Entre las actividades de autoconsumo que se pueden destacar, está la crianza de animales, cultivos de huerto, productos de conserva y elaboración de prendas. Las actividades de trabajo no remunerado de apoyo a otros hogares y a la comunidad hacen referencia a la participación voluntaria en obras 0 actividades de la comunidad, incluyendo las religiosas.

La tasa de participación ${ }^{7}$ del trabajo remunerado indica que el 54,91 \% está incorporada en el mercado de trabajo, con una dedicación promedio de tiempo aproximada a 34 horas, de las cuales, en su mayoría, corresponde a su ocupación principal. También es importante señalar, que el 40,37 \% se debe desplazar desde su hogar al lugar de trabajo, dedicando un poco más de tres horas.

\section{Actividades de no trabajo}

En relación con las actividades de no trabajo, solamente un 5,28 \% de las mujeres rurales jefas de hogar dedican tiempo a actividades de formación, con una dedicación promedio de 17:28 horas semanales. Básicamente, se trata del tiempo utilizado para trasladarse y asistir a clases, así como para estudiar y hacer tareas. En cuanto al uso del tiempo para necesidades personales, en promedio dedican 72:07 horas, de las cuales, en su mayoría, están destinadas a dormir, comer y ciertos cuidados de índoles personales. Por otra parte, la participación en cursos a través de internet o distancia, no llega al $1 \%$.

En lo que corresponde al tiempo libre, prácticamente todas realizan algún tipo de actividad, con un promedio cercano a las 30 horas semanales. El detalle de los datos y respectivo análisis de las actividades de tiempo libre se presenta en el siguiente apartado.

\footnotetext{
${ }^{6}$ Promedio de horas semanales dedicadas a determinada actividad por parte de la población que reportó realizarla.

${ }^{7}$ La tasa de participación hace referencia al porcentaje de la población de 12 años y más que dedicó tiempo a determinada actividad.
} 
Actividades de tiempo libre

De acuerdo con el INEC ${ }^{8}$, las actividades de tiempo libre hacen referencia a compartir actividades con los demás miembros de su hogar, tales como: ver televisión, escuchar música, jugar ajedrez, visitar parques, museos, asistir a cumpleaños, fiestas patronales, bautizos, conversar o enviar mensajes por teléfono y otras. En específico, esta categoría abarca cinco actividades: medios masivos de comunicación, convivencia familiar, social y participación ciudadana, participación en juegos, aficiones y visita a lugares de entretenimiento, práctica de deportes y ejercicio físico y ocio pasivo. Como se observa en el cuadro 7, las mujeres rurales jefas de hogar en su mayoría dedican una gran parte de su tiempo libre a ver televisión y utilizar su teléfono celular.

\section{Cuadro 6}

Costa Rica: Costa Rica: Mujeres rurales jefas de hogar. Actividades de no trabajo. Tasa de participación y tiempo efectivo promedio

\begin{tabular}{|l|c|c|}
\hline Actividades de no trabajo & $\begin{array}{c}\text { Tasa de } \\
\text { participa- } \\
\text { ción }\end{array}$ & $\begin{array}{c}\text { Tiempo } \\
\text { efectivo } \\
\text { promedio }\end{array}$ \\
\hline Actividades de formación & $\mathbf{5 , 2 8}$ & $\mathbf{1 7 : 1 7}$ \\
\hline Asistir a clases & 2,72 & $14: 19$ \\
\hline Curso presencial & 1,52 & $4: 38$ \\
\hline Curso internet o distancia & 0,88 & $8: 18$ \\
\hline Estudiar, hacer tareas, otras & 3,50 & $6: 14$ \\
\hline Horas beca, estudiante u otras & 0,14 & $12: 00$ \\
\hline Traslado a actividades educativas & 4,41 & $3: 15$ \\
\hline Necesidades personales & 100,00 & $72: 18$ \\
\hline Dormir & 100,00 & $56: 04$ \\
\hline Comer & 100,00 & $7: 43$ \\
\hline Necesidades y cuidados personales & 100,00 & $7: 04$ \\
\hline Asistir a cuidado de cabello, uñas, otros & 9,65 & $1: 10$ \\
\hline Consulta médica o autoexámenes & 16,74 & $3: 22$ \\
\hline Recibió terapias, rehabilitación & 0,43 & $4: 09$ \\
\hline Recuperación de malestar o enfermedad & 12,43 & $6: 13$ \\
\hline & & \\
\hline Tiempo libre & $\mathbf{9 9 , 9 2}$ & $\mathbf{2 9 : 3 4}$ \\
\hline
\end{tabular}

Fuente: INEC, Costa Rica. Encuesta Nacional de Uso del Tiempo, 2017.

8 INEC, Encuesta Nacional de..., 23. 
Casi un $95 \%$ de las mujeres participa en actividades de convivencia familiar, social y participación ciudadana, principalmente, conversar cara a cara con personas de su propio hogar, visitar o atender personas o asistir a fiestas, bautizos, funerales $u$ otras, así como participar en actividades religiosas o espirituales. Por su parte, la participación y tiempo dedicado a celebraciones comunales o asistencia a celebraciones cívicas o políticas resulta muy baja. Algo similar ocurre con la participación en juegos, aficiones y visita a lugares de entretenimiento, solamente un 10,76 \% dedica tiempo a actividades artísticas, de colección, juegos, cursos libres o visitar lugares de entretenimiento. Solamente una quinta parte dedica tiempo a la práctica de deportes y ejercicio físico, con un promedio cercano a las tres horas. En relación con el ocio pasivo, que trata de si la persona está acostada, sentada, pensando o reflexionando sin hacer ninguna otra actividad, un 66,18 \% dedica algún tiempo a estas actividades con un promedio de tiempo de casi siete horas.

\section{Brechas en el uso del tiempo entre las jefaturas de hogar en la zona rural}

En el presente apartado, con el fin de conocer y cuantificar las brechas que se establecen en el uso del tiempo entre las mujeres y los hombres jefes de hogar de la zona rural, se examina el tiempo efectivo promedio que ambos dedican a las actividades de no trabajo y de trabajo, así como la carga global de trabajo, según grupo de edad, estado conyugal, nivel de instrucción y posición en el empleo.

El cuadro 8 contiene información referente al tiempo efectivo promedio en actividades de trabajo y de no trabajo, presta atención a las actividades de trabajo doméstico no remunerado. Justamente, como se puede observar, las mayores diferencias se establecen en las actividades de trabajo doméstico no remunerado, en donde el tiempo promedio efectivo que destinan las mujeres es más del doble que el tiempo destinado por los hombres. Mientras las mujeres le dedican casi 43 horas, los hombres destinan cerca de 16 horas, es decir, una diferencia de 27 horas. Estas diferencias se reflejan en las actividades relacionadas con la preparación y servicio de alimentos y bebidas, limpieza y mantenimiento de la vivienda y limpieza y cuidado de ropa y calzado, así como en algunas actividades de cuido, principalmente, el cuido de personas dependientes mayores a 12 años.

En el caso de las actividades relacionadas con el trabajo de autoconsumo, los hombres le dedican más tiempo, casi el doble, lo cual es frecuente en la zona rural, considerando que se tratan de actividades relacionadas principalmente con la cría de animales, cultivos huertos y reparaciones mayores a la vivienda. En referencia al trabajo no remunerado de apoyo a otros hogares y a la comunidad, la diferencia es más de una hora a favor de los hombres.

En un sentido contrario a las actividades de trabajo no remunerado, se direcciona el tiempo destinado a las actividades de trabajo remunerado. Como se puede observar en el cuadro 8 , mientras que las mujeres le dedican un 


\section{Cuadro 7}

Costa Rica: Mujeres rurales jefas de hogar. Actividades de tiempo libre. Tasa de participación y tiempo efectivo promedio

\begin{tabular}{|l|c|c|}
\hline Actividades de tiempo libre & $\begin{array}{c}\text { Tasa de } \\
\text { partici- } \\
\text { pación }\end{array}$ & $\begin{array}{c}\text { Tiempo } \\
\text { efectivo } \\
\text { promedio }\end{array}$ \\
\hline Uso de medios masivos de comunicación & $\mathbf{9 5 , 5 5}$ & $\mathbf{1 5 : 0 2}$ \\
\hline Ver programas de TV, videos, series y otros & 88,84 & $10: 54$ \\
\hline Escuchar música, noticias u otros & 19,91 & $6: 16$ \\
\hline $\begin{array}{l}\text { Revisar redes sociales, correo, hablar por teléfono, } \\
\text { chatear }\end{array}$ & 64,65 & $4: 17$ \\
\hline $\begin{array}{l}\text { Consultar información por internet, leyó libro u } \\
\text { otro material digital }\end{array}$ & 8,91 & $1: 58$ \\
\hline Leer impresos & 15,54 & $3: 11$ \\
\hline $\begin{array}{l}\text { Convivencia familiar, social y participación } \\
\text { ciudadana }\end{array}$ & $\mathbf{9 4 , 1 7}$ & $\mathbf{9 : 4 4}$ \\
\hline Conversar cara a cara personas hogar & 66,22 & $4: 52$ \\
\hline $\begin{array}{l}\text { Visitar o atender personas o asistió a fiestas, } \\
\text { bautizos, funerales u otras }\end{array}$ & 49,87 & $6: 30$ \\
\hline Participar en actividades religiosas o espirituales & 70,94 & $3: 42$ \\
\hline Participar celebraciones comunales & 2,49 & $2: 53$ \\
\hline Asistir celebraciones cívicas o políticas & 0,07 & $3: 00$ \\
\hline & & \\
\hline Participación en juegos, aficiones y visita a & $\mathbf{1 0 , 7 6}$ & $\mathbf{7 : 0 7}$ \\
\hline lugares de entretenimiento & $\mathbf{1 , 1 7}$ & $12: 59$ \\
\hline $\begin{array}{l}\text { Realizar actividades artísticas (no por trabajo o } \\
\text { estudio) }\end{array}$ & $\mathbf{2 0 , 7 4}$ & $3: 30$ \\
\hline Coleccionar objetos u otros & 3,20 & $5: 48$ \\
\hline Jugar, hacer rompecabezas u otros & 1,46 & $15: 03$ \\
\hline Llevar cursos por afición & 5,03 & $3: 38$ \\
\hline Visitar lugares de entretenimiento & $\mathbf{2 0 , 4 4}$ & $\mathbf{3 : 1 2}$ \\
\hline Práctica de deportes y ejercicio físico & $\mathbf{6 : 5 6}$ \\
\hline Ocio pasivo & & \\
\hline & & \\
\hline
\end{tabular}

Fuente: INEC, Costa Rica. Encuesta Nacional de Uso del Tiempo, 2017. 


\section{Cuadro 8}

Costa Rica: Mujeres y hombres jefes de hogar de la zona rural. Tiempo efectivo promedio en actividades de trabajo y de no trabajo

\section{Actividades}

Actividades de trabajo

Trabajo doméstico no remunerado

Preparación y servicio de alimentos y bebidas

Limpieza y mantenimiento de la vivienda

Construcción y reparaciones menores de la vivienda y vehículo

Limpieza y cuidado de ropa y calzado

Compras del hogar

Gerencia y administración del hogar

Cuidados de salud de menores de 12 años

Cuido de personas del hogar de 12 años y más

Cuido personas hogar totalmente dependientes

12 años y más

Trabajo de autoconsumo

4:41

$8: 41$

Trabajo no remunerado de apoyo a otros hogares y a la comunidad

Trabajo remunerado

33:55

51:52

Trabajo en ocupación principal

$31: 20$

46:55

Trabajo en ocupación secundaria

$10: 31$

16:08

\section{Actividades de no trabajo}

Actividades de formación

17:17

16:08

Necesidades personales

72:18

70:35

\section{Tiempo libre}

Uso de medios masivos de comunicación

29:34

33:18

Práctica de deportes y ejercicio físico

15:02

$17: 15$

Ocio pasivo

$3: 12$

$4: 48$

$6: 56$

$8: 29$

Fuente: INEC, Costa Rica. Encuesta Nacional de Uso del Tiempo, 2017. 
promedio cercano a las 34 horas, los hombres destinan casi 52 horas, casi una diferencia de 18 horas. Dicha diferencia se refleja tanto en la ocupación principal como en la ocupación secundaria.

En actividades de no trabajo, como formación, necesidades personales y tiempo libre, no se establecen diferencias por destacar. No obstante, en el caso particular de las actividades de tiempo libre relacionadas con el uso de medios masivos de comunicación, la práctica de deportes y ejercicio físico, así como el ocio pasivo, los hombres dedican un poco más de tiempo. Todas estas diferencias se reflejan en cálculo de las respectivas cargas globales de trabajo que se presentan en el cuadro 9.

De acuerdo con el INEC ${ }^{9}$, la carga global de trabajo contempla el tiempo efectivo utilizado en trabajo doméstico no remunerado y el trabajo remunerado, lo cual refleja la magnitud de los dos tipos de trabajo que recae sobre uno u otro sexo. En específico, en este contexto y dadas las mayores diferencias en el tiempo efectivo destinado al trabajo doméstico no remunerado que se mostraron en el anterior apartado, la carga global de trabajo es más alta para las mujeres. Como se observa en el cuadro 9, la cual contiene la información correspondiente a la carga global de trabajo para las mujeres y hombres, es clara la diferencia que se establece entre uno y otro, en cualquiera de los rubros en consideración.

En términos generales, la diferencia en la carga global de trabajo es de más de 8 horas. En el caso particular de los grupos de edad, la mayor carga global de trabajo recae en las mujeres menores de 35 años, aunque se debe reconocer que la diferencia con los otros grupos de edad de mujeres no resulta del todo muy amplia. En lo que corresponde al estado conyugal, la mayor carga global de trabajo se refleja en el grupo de mujeres divorciadas, separadas o viudas. A nivel de instrucción, las mujeres con estudios de secundaria completa o superior son las que registran la mayor carga global de trabajo, con cifras superiores a las 75 horas, incluso cerca de las 83 horas en el caso de las mujeres con estudios de secundaria. En cuanto a la posición en el empleo, las mujeres empleadas en una empresa o institución son las que muestran una mayor carga global de trabajo, con casi 12 más que un hombre en su misma posición. Algo similar ocurre con las mujeres que trabajan por cuenta propia, donde la diferencia en la carga global de trabajo es de 11 horas.

\section{Conclusiones}

En definitiva, los datos sobre el uso del tiempo constituyen una importante herramienta para reconocer y comprender la participación de la mujer en la fuerza laboral, tanto remunerada como no remunerada. En el caso particular de Costa Rica, los resultados de la ENUT 2017 evidencian que las brechas de

9 INEC, Encuesta Nacional de..., 51. 


\section{Cuadro 9}

Costa Rica: Mujeres y hombres rurales jefes de hogar. Carga global de trabajo según grupo de edad, estado conyugal, nivel de instrucción y posición en el empleo

\begin{tabular}{|c|c|c|}
\hline Característica & Mujeres & Hombres \\
\hline Total & $71: 43$ & $63: 25$ \\
\hline \multicolumn{3}{|l|}{ Grupos de edad } \\
\hline 12 a 19 años & $92: 47$ & $64: 24$ \\
\hline 20 a 35 años & $76: 00$ & $70: 30$ \\
\hline 36 a 54 años & $72: 21$ & $64: 22$ \\
\hline 55 y más años & $73: 44$ & $55: 56$ \\
\hline \multicolumn{3}{|l|}{ Estado conyugal } \\
\hline Casadas(os) o unidas(os) & $71: 53$ & $63: 26$ \\
\hline Divorciadas(os), separadas(os) o viudas(os) & $76: 25$ & 69:32 \\
\hline Solteras(os) & 74:06 & $68: 03$ \\
\hline \multicolumn{3}{|l|}{ Nivel de instrucción } \\
\hline Primaria incompleta y menos & 73:04 & $61: 40$ \\
\hline Primaria completa y secundaria incompleta & $73: 25$ & $65: 02$ \\
\hline Secundaria completa & $82: 47$ & $65: 20$ \\
\hline Superior & $77: 22$ & $68: 37$ \\
\hline \multicolumn{3}{|l|}{ Posición en el empleo } \\
\hline Un negocio o actividad propia & 75:01 & 64:07 \\
\hline $\begin{array}{l}\text { Como empleada o empleado de una } \\
\text { persona/empresa o institución }\end{array}$ & $76: 47$ & $64: 47$ \\
\hline Como empleada o empleado de casas particulares & 69:46 & $56: 14$ \\
\hline $\begin{array}{l}\text { Como ayuda a una persona familiar o conocido que } \\
\text { tiene un negocio o actividad propia }\end{array}$ & 75:11 & $64: 04$ \\
\hline $\begin{array}{l}\text { Como ayuda a una persona o familiar conocido que } \\
\text { trabaja para un patrón o empresa }\end{array}$ & $63: 16$ & $85: 35$ \\
\hline Necesidades personales & 72:18 & 70:35 \\
\hline Tiempo libre & 29:34 & 33:18 \\
\hline Uso de medios masivos de comunicación & 15:02 & $17: 15$ \\
\hline Práctica de deportes y ejercicio físico & $3: 12$ & $4: 48$ \\
\hline Ocio pasivo & $6: 56$ & $8: 29$ \\
\hline
\end{tabular}

Fuente: INEC, Costa Rica. Encuesta Nacional de Uso del Tiempo, 2017. 
género en el uso del tiempo siguen mediadas por la cantidad de tiempo empleado en la realización del trabajo doméstico no remunerado. Particularmente en el contexto del medio rural, aunque actualmente las mujeres cuentan con mejores alternativas de inserción al mercado laboral y acceso a la educación, los datos de la ENUT 2017 muestran que siguen concentrando, con un gran margen de diferencia, las actividades domésticas.

Entre las principales características de las mujeres rurales jefas de hogar, a la luz de la ENUT 2017, se puede destacar que cerca del $45 \%$ de ellas se encuentra separadas o solteras, muy por encima del 32,9\% que conviven en unión. En cuanto a su nivel de instrucción, un poco más del $80 \%$ apenas cuentan con primaria o secundaria incompleta, donde solamente el 7,53\% tienen secundaria completa y el 10,12 \% cuentan con algún grado de educación superior. También, se determinó que al momento de la encuesta, más del $90 \%$ no da continuidad a sus estudios debido a situaciones como embarazo, trabajo o por las propias actividades que demanda el hogar. Respecto de sus condiciones de empleo, casi el $80 \%$ corresponde a ocupaciones no elementales y trabajadoras de los servicios y vendedoras de comercios y mercados, llevando a cabo su actividad laboral por cuenta propia o como empleada de una persona, empresa, institución o en casas particulares.

Al estudiar las actividades que conforman el trabajo doméstico no remunerado, se determinó que las mujeres rurales jefas de hogar le dedican un tiempo efectivo promedio cercano a las 43 horas semanales, en actividades propiamente relacionadas con la limpieza y el mantenimiento de la vivienda, la preparación de alimentos y el cuidado de menores. La tasa de participación del trabajo remunerado indica que el $54,91 \%$ de las mujeres rurales jefas de hogar están incorporadas en el mercado de trabajo, con una dedicación promedio de tiempo aproximada a 34 horas semanales. En lo que corresponde al tiempo libre, le dedican un promedio cercano a las 30 horas semanales, en su mayoría destinado a ver televisión y utilizar su teléfono celular. Solamente una quinta parte dedican tiempo a la práctica de deportes y ejercicio físico, con un promedio cercano a las tres horas. En relación con el ocio pasivo, un 66,18\% dedica algún tiempo a estas actividades, con un promedio de tiempo de casi siete horas semanales.

De igual forma se determinó que mientras las mujeres rurales jefas de hogar le dedican casi 43 horas semanales a las actividades de trabajo no remunerado, los hombres rurales jefes de hogar le dedican 16 horas semanales, es decir, una diferencia de 27 horas. En cuanto al tiempo destinado a las actividades de trabajo remunerado, las mujeres rurales jefas de hogar le dedican un promedio cercano a las 34 horas semanales y los hombres rurales jefes de hogar casi 52 horas semanales, una diferencia de 18 horas. Esto anterior se refleja en una mayor carga global de trabajo para las mujeres, en particular las que tienen menos de 35 años, que no tienen pareja y cuentan con estudios de secundaria.

Si duda, resulta importante volver a mirar estos datos a luz de la nueva realidad que plantea la pandemia debido a la COVID-19, considerando los cambios en las 
cargas globales de trabajo de las mujeres, principalmente las repercusiones que trae consigo el teletrabajo, las clases virtuales, las medidas de confinamiento, el desempleo y la consecuente reducción de ingresos monetarios en el ámbito de los hogares.

A manera de recomendación, es necesario promover alternativas que potencien la utilización de los datos que arrojan estos tipos de encuestas, ya que no solamente constituyen un esfuerzo de tiempo y de recursos económicos por parte del Estado que se deben aprovechar, sino que también se convierten en una herramienta objetiva y válida para reflejar situaciones que ocurren en nuestra sociedad y que son constantemente invisibilizadas.

\section{Formato de citación según APA}

Porras-Solís, Á. J. (2021). Uso del tiempo de las mujeres rurales jefas de hogar en Costa Rica. Revista Espiga, 20(42), 169-187.

Formato de citación según Chicago-Deusto

Porras-Solís, Ángel Jesús. «Uso del tiempo de las mujeres rurales jefas de hogar en Costa Rica». Revista Espiga 20, n.o 42 (julio - diciembre, 2021): 169-187. 


\section{Referencias}

Bárcena, Alicia. «Mujeres rurales y Agenda 2030 en América Latina y el Caribe: una mirada desde el mercado de trabajo». En: Luchadoras: mujeres rurales en el mundo: 28 voces autorizadas, 21-26, San José: IICA, 2018. Acceso: 15 de enero de 2021, https://repositorio.iica.int/bitstream/handle/ 11324/7221/BVE18040310e. pdf?sequence=1\&isAllowed=y

INEC. Encuesta Nacional de Uso del Tiempo 2017: Resultados generales. San José: INEC, 2018. Acceso: 12 de julio de 2021. https://www.inec.cr/sites/ default/files/documetos-biblioteca-virtual/reenut2017.pdf

Navarro-Ochoa, Angélica. «¿Mujeres proveedoras y jefas de familia? Nuevas realidades rurales en localidades de la región zamorana». La ventana. Revista de estudios de género 4, n. ${ }^{\circ} 31$ (2010): 139-171. Acceso: 25 de enero de 2021. http://www.scielo.org.mx/scielo.php? script=sci_arttext\&pid=S1405-94362010000100007\&lng=es\&tIng=es

Sandoval-Carvajal, Irma y González-Vega, Lidia María. «Estimación del valor económico del trabajo no remunerado en Costa Rica. Resultados e ilustración metodológica». Estudios Demográficos y Urbanos 30, n. ${ }^{\circ} 3$, (2015): 691-724. Acceso: 02 de febrero de 2021. https:// estudiosdemograficosyurbanos.colmex.mx/index.php/edu/article/view/ $1497 / 1490$

Waring, Marilyn. «Política y estrategia de provisión de la atención de salud en la región de la OPS y medición del trabajo no remunerado». En: La economía invisible y las desigualdades de género. La importancia de medir y valorar el trabajo no remunerado, 245-286. Washington D.C.: OPS, 2008. Acceso: 15 de enero de 2021. https://iris.paho.org/bitstream/handle/10665.2/6034/ LA\%20ECONOMIA\%20INVISIBLE. pdf?sequence=1\&isAllowed=y 